\title{
LARGE-SCALE CANDIDATE GENE STUDY OF TUBERCULOSIS SUSCEPTIBILITY IN THE KARONGA DISTRICT OF NORTHERN MALAWI
}

\author{
JODENE FITNESS, SIAN FLOYD, DAVID K. WARNDORFF, LIFTED SICHALI, SIMON MALEMA, \\ AMELIA C. CRAMPIN, PAUL E. M. FINE, AND ADRIAN V. S. HILL \\ Wellcome Trust Centre for Human Genetics, University of Oxford, Oxford, United Kingdom; Department of Tropical Hygiene, \\ London School of Hygiene and Tropical Medicine, London, United Kingdom; Karonga Prevention Study, \\ Chilumba, Karonga District, Malawi
}

\begin{abstract}
Twenty-seven polymorphisms from 12 genes have been investigated for association with tuberculosis (TB) in up to 514 cases and 913 controls from Karonga district, northern Malawi. Homozygosity for the complement receptor 1 (CR1) Q1022H polymorphism was associated with susceptibility to TB in this population (odds ratio [OR] $=3.12,95 \%$ Confidence interval $[\mathrm{CI}]=1.13-8.60, P=0.028$ ). This association was not observed among human immunodeficiency virus (HIV)-positive TB cases, suggesting either chance association or that HIV status may influence genetic associations with TB susceptibility. Heterozygosity for a newly studied CAAA insertion/deletion polymorphism in the 3'untranslated region of solute carrier family 11, member 1 (SLC11A1, formerly NRAMP1) was associated with protection against TB in both HIV-positive $(\mathrm{OR}=0.70,95 \% \mathrm{CI}=0.49-0.99, P=0.046)$ and $\mathrm{HIV}$-negative $(\mathrm{OR}=0.65,95 \% \mathrm{CI}$ $=0.46-0.92, P=0.014)$ TB cases, suggesting that the SLC11A1 protein may have a role in innate TB immune responses that influence susceptibility even in immunocompromised individuals. However, associations of other variants of SCLA11A with TB reported from other populations were not replicated in Malawi. Furthermore, associations with vitamin $\mathrm{D}$ receptor, interferon- $\gamma$, and mannose-binding lectin observed elsewhere were not observed in this Karonga study. Genetic susceptibility to TB in Africans appears polygenic. The relevant genes and variants may vary significantly between populations, and may be affected by HIV infection status.
\end{abstract}

\section{INTRODUCTION}

Approximately eight million new cases and almost 1.8 million deaths attributable to tuberculosis (TB) occur each year. ${ }^{1}$ In many populations, this situation is exacerbated by an increasing prevalence of infection with human immunodeficiency virus (HIV). Tuberculosis is caused by Mycobacterium tuberculosis, an intracellular pathogen that survives within macrophages. It is primarily a pulmonary disease, but it may affect organs other than the lungs, and it has been estimated that only $10 \%$ of infected individuals develop clinical disease. ${ }^{2}$ Evidence from family, twin, and case-control studies indicates that host genetic factors play a role in determining the outcome of exposure and infection. ${ }^{3}$

The first convincing evidence related to HLA genes: the HLA-DR2 molecule is associated with TB susceptibility in some populations, while DQ1 alleles are associated in others. ${ }^{4}$ Non-HLA genes have also been implicated in TB susceptibility. Variants of solute carrier family 11, member 1 (SLC11A1, formerly NRAMP1) have been associated with TB susceptibility in populations from The Gambia, GuineaConakry, Korea, Brazil and Japan. ${ }^{5-9}$ Also, evidence for linkage of a TB susceptibility locus to a $2 \mathrm{q} 35$ region that contains SLC11A1 has been reported in a Canadian Aboriginal pedigree. ${ }^{10} \mathrm{~A}$ variant in the vitamin $\mathrm{D}$ receptor (VDR) has been associated with TB susceptibility in The Gambia. ${ }^{11}$ There is limited and conflicting evidence suggesting that variants in mannose-binding lectin (MBL2) may be associated with either protection or susceptibility to TB. ${ }^{12,13}$ Recently, an interferon- $\gamma$ (IFNG) polymorphism (+874 T/A) that may cause increased IFNG transcription in response to $\mathrm{NF}-\kappa \mathrm{B}$ has been found to be associated with resistance to TB in Sicilians and South Africans. ${ }^{14-16}$

The Karonga Prevention Study (KPS), a long-term epidemiologic study of mycobacterial disease in the Karonga District of northern Malawi, provides an ideal opportunity to evaluate genetic associations with TB that have been reported from other populations, as well as to generate and investigate new hypotheses. In recognition of this, both case control and multicase family studies have been set up in this population to investigate genetic risk factors for both TB and leprosy. This paper reports on the TB case-control study. Given that HIV is a major risk factor for TB in this population, as elsewhere in southern Africa, and the possibility that the influence of some genetic factors may depend upon the level of immune competence of the host, both HIV-negative and HIV-positive TB patients were studied.

Twenty-seven non-HLA polymorphisms from 12 genes were investigated for association with TB susceptibility in this population. Variants in SCL11A1, VDR, MBL2, and IFNG were included because these genes have been associated with TB susceptibility in other populations. Interleukin-10 (IL10) is a key anti-inflammatory cytokine that has recently been implicated in leprosy susceptibility in Brazil. ${ }^{17}$ Polymorphisms in tumor necrosis factor (TNF, formerly $\mathrm{TNF} \alpha$ ), and lymphotoxin alpha (LTA, formerly TNF $\beta$ ) have been associated with leprosy susceptibility. The toll-like receptors 2 and 4 (TLR2 and TLR4) are pattern recognition molecules involved in both innate and adaptive immune responses. Variants of toll receptors have recently been associated with leprosy, malaria and pneumoccocal disease (Cooke G, Aucan C, Walley AJ, Segal S and others, unpublished data). ${ }^{18}$ The chemokine (C-C motif) ligand 3 (CCL3, formerly MIP1 $\alpha$ ) was selected as a functional candidate due to its involvement in $\mathrm{T}$ helper 1 cell (TH1) differentiation. A variant in intracellular adhesion molecule 1 (ICAM1) may be associated with susceptibility to severe malaria. ${ }^{17}$ Finally, since complement receptor 1 (CR1) can be used by M. tuberculosis to gain entry into macrophages, it was hypothesized that some recently defined variants in CR1 may influence susceptibility to mycobacterial disease. ${ }^{17}$

\section{MATERIALS AND METHODS}

The study protocol was reviewed and approved by the National Health Sciences Research Committee of Malawi and by 
the Ethics Committee of the London School of Hygiene and Tropical Medicine.

Recruitment and sample collection. The population, basic field methods, and TB case finding and diagnostic procedures of the KPS have been described in detail elsewhere. ${ }^{19,20} \mathrm{Tu}$ berculosis cases recruited into the study were ascertained largely by passive self-reporting at health centers, although a small proportion were identified in the context of other studies. Sputum samples and lymph node aspirates were examined (fluorescence and Ziehl-Neelsen stain) and cultured at project headquarters in Chilumba, with species confirmation by the United Kingdom Public Health Laboratory Service Mycobacterial Reference Unit (Dulwich, United Kingdom). Biopsy specimens were sent to Professor S. Lucas (St. Thomas' Hospital, London, United Kingdom). Inclusion in this study required confirmation of TB by culture, smear, or histology (excluding those whose only evidence of TB was a single scanty smear with fewer than 10 bacilli).

Recruitment for this study began in 1996, with targets of 250 HIV-positive and 250 HIV-negative cases. Cases diagnosed from November 1996, together with cases diagnosed before this and known to be HIV-negative at the time of diagnosis (as all TB cases in Karonga have been offered HIV counseling and testing since 1988), were included. For cases diagnosed before 1998, up to four individually matched controls were selected using project databases. Individuals were eligible for selection as controls if they had no history or evidence of either TB or leprosy and they were not a first- or second-degree relative of the case to which they were being matched. Matching criteria were age (within age groups 15-19, 20-24, 25-29, 30-34, 35-44, 45-54, 55-64, and $\geq 65$ years old), sex, and area of residence (within $1 \mathrm{~km}$ of the case). From 1998 a field-based method was used to identify controls frequency-matched to cases for age (using date of birth in 10-year calendar periods), sex, and area of residence. ${ }^{21}$ Approximately two controls were recruited for each case. Analyses reported here include cases and controls recruited up to the end of September 2001.

Cases and controls were interviewed by project paramedical staff who explained the study, provided counseling, and invited the patients to provide a blood sample for HIV testing and genetic analyses. Blood $(7.5 \mathrm{~mL})$ for HIV and genetics testing was collected into EDTA, and placed in vaccine carriers with wet ice until it reached the project laboratory within three days of collection, where cells were separated and stored at $-70^{\circ} \mathrm{C}$. The DNA was separated with Nucleon kits (Scotlab, Coatbridge, United Kingdom) following the manufacturer's instructions, and shipped at $4^{\circ} \mathrm{C}$ to the Wellcome Trust Centre for Human Genetics (Oxford, United Kingdom) for analysis. Those who wished to know their HIV status were given post-test counseling in their homes. The HIV testing was done in duplicate using a particle agglutination test (Edgware modification of Serodia; Mast Diagnositcs, Ltd., Bootle, Merseyside, United Kingdom) and an ELISA (Vironostika HIV Uni-form II plus 0; Organon Teknika, Ltd., Cambridge, United Kingdom), with retesting of discordant samples. ${ }^{22}$

Genotyping. The methods used for genotyping the 27 variants investigated have been described elsewhere. ${ }^{17}$ The ligation detection reaction was used to type polymorphisms in VDR, MBL, IL10, TNF, ICAM1, CR1, and TLR4. ${ }^{17}$ The SLC11A1, CCL3, TLR2, and LTA polymorphisms were typed by fluorescence polymerase chain reaction (PCR) and an amplification refractory mutation system $\mathrm{PCR}^{17}$ was used to type the IFNG variant.

Statistical analysis. Analysis of the individually-matched and frequency-matched studies was done separately. For the individually-matched studies, conditional logistic regression was used to quantify the association between disease and genotype, with and without controlling for ethnic group. For the frequency-matched study, logistic regression was used. All analyses were stratified on HIV status of the case.

In general, HIV-negative controls were used as the comparison group for both HIV-negative and HIV-positive cases. For HIV-negative cases, HIV-negative individuals are the most valid control group. For HIV-positive cases, HIVpositive individuals are the ideal control group. However, relatively few HIV-positive controls were identified, and restricting the control group to them would considerably limit study power. If the genes studied are not related to the risk of HIV infection, then it is acceptable to use HIV-negative individuals, rather than HIV-positive individuals, as the control group for HIV-positive cases. The association between genotype and HIV status was thus assessed among the controls. For the polymorphisms where there was preliminary evidence of an association, HIV-positive controls were used as the comparison group for HIV-positive cases.

Two estimates of effect (odds ratios [OR]) were obtained for each polymorphism, one from the individually matched study and one from the frequency-matched study. A summary estimate was obtained by combining the two estimates in a meta-analysis.

Genotyping was not always successful, and for some polymorphisms genotyping was attempted on only a proportion of individuals. Thus, the number of individuals genotyped was less than the total number of individuals who gave blood for genetics and HIV testing.

\section{RESULTS}

Four hundred and thirty-one individuals were recruited into the individually matched study (133 cases and 298 controls). The HIV status was known for all individuals except for one control, and DNA was obtained from 418 individuals. A total of 1,589 individuals were recruited into the frequencymatched case-control study (546 cases and 1,043 controls). Of these individuals, 1,132 provided blood for testing (412 cases and 720 controls), and 174 controls and 1 case provided a buccal swab. Genetic data were analyzed for slightly different numbers of individuals for each gene up to a total of 1,188 individuals. The HIV data were available for $85 \%$ of these individuals; thus, 1,010 individuals (386 cases and 624 controls) were included in the analyses.

Preliminary comparisons of genotype frequencies between HIV-positive controls $(\mathrm{n}=82)$ and HIV-negative controls $(\mathrm{n}=542)$ indicated that the polymorphisms TNF-238, VDRBsm I, and CR1-4795 may be associated with HIV in Malawians (compare the controls in Tables 1 and 2). In each case the rarest genotype showed a weak association $(P=0.01$, 0.02 , and 0.04 , respectively). Thus, for these polymorphisms, HIV-positive TB cases were compared with HIV-positive controls to assess the association between TB and genotype (Table 1). Homozygosity for the Bsm I polymorphism in VDR appeared to provide protection against TB among HIV- 
TABLE 1

Genotype frequencies and odds ratios (ORs) for HIV-positive cases and HIV-positive controls*

\begin{tabular}{|c|c|c|c|c|c|c|c|}
\hline \multirow[b]{2}{*}{ Gene } & \multirow[b]{2}{*}{ Polymorphism } & \multirow[b]{2}{*}{ Genotype } & \multicolumn{2}{|c|}{$\mathrm{HIV}+$ cases } & \multicolumn{2}{|c|}{$\mathrm{HIV}+$ controls } & \multirow[b]{2}{*}{$\begin{array}{c}\text { HIV+ cases compared to HIV+ controls OR ( } 95 \% \text { confidence limits) } \\
P \text { values adjusted for age, sex, zone, ethic group }\end{array}$} \\
\hline & & & $\%$ & $n$ & $\%$ & $\mathrm{n}$ & \\
\hline \multirow[t]{4}{*}{ CR1 } & $\mathrm{A} \rightarrow \mathrm{G}$ at 4795 & $\mathrm{a} / \mathrm{a}$ & 64 & 161 & 68 & 52 & \\
\hline & K1590E & $\mathrm{a} / \mathrm{g}$ & 30 & 75 & 32 & 24 & $1.06(0.59-1.92) 0.837$ \\
\hline & $\left(\mathrm{McC}^{\mathrm{a}} \rightarrow \mathrm{McC}^{\mathrm{b}}\right)$ & $\mathrm{g} / \mathrm{g}$ & 6 & 16 & 0 & 0 & \\
\hline & & & & 252 & & 76 & \\
\hline \multirow[t]{4}{*}{ TNF } & $-238(\mathrm{G} \rightarrow \mathrm{A})$ & $g / g$ & 85 & 122 & 88 & 43 & \\
\hline & & $\mathrm{g} / \mathrm{a}$ & 15 & 21 & 8 & 4 & $2.01(0.65-6.28) 0.228$ \\
\hline & & $\mathrm{a} / \mathrm{a}$ & 0.7 & 1 & 4 & 2 & $0.11(0.005-2.58) 0.171$ \\
\hline & & & & 144 & & 49 & \\
\hline \multirow[t]{4}{*}{ VDR } & $\mathrm{C} \rightarrow \mathrm{T}$ & $\mathrm{c} / \mathrm{c}$ & 62 & 123 & 60 & 36 & \\
\hline & $(B s m \mathrm{I})$ & $\mathrm{c} / \mathrm{t}$ & 35 & 69 & 23 & 14 & $1.65(0.78-3.52) 0.194$ \\
\hline & $(b \rightarrow B)^{\prime}$ & $\mathrm{t} / \mathrm{t}$ & 3 & 6 & 17 & 10 & $0.12(0.04-0.40) 0.001$ \\
\hline & & & & 198 & & 60 & \\
\hline
\end{tabular}

TNF = tumor necrosis factor; VDR = vitamin D receptor

infected individuals $(\mathrm{OR}=0.12,95 \% \mathrm{CI}=0.04-0.4, P=$ $0.001)$. However, this association results from a high frequency of the rarer homozygote $(17 \%)$ in the small $(n=60)$ HIV-positive control group and could be a chance finding. There was no evidence that the TNF-238 and CR1-4795 variants were associated with TB in HIV-infected individuals.

The 24 polymorphisms that did not show evidence for association with HIV were tested for association with TB by comparing genotype frequencies of both HIV-negative and HIV-positive cases against those of HIV-negative controls. Results are shown in Table 2. Among HIV-negative individuals, homozygosity for the CR1 variant Q1022H was associated with $\mathrm{TB}(\mathrm{OR}=3.12,95 \% \mathrm{CI}=1.13-8.60, P=0.028)$. Evidence for association with this variant was not found among HIV-positive TB cases.

On comparing HIV-positive TB cases with HIV-negative controls, associations were observed with MBL2 G57E heterozygotes $(\mathrm{OR}=1.69,95 \% \mathrm{CI}=1.04-2.75, P=0.034)$, and IL10-1082 G homozygotes ( OR $=0.37,95 \% \mathrm{CI}=0.18-0.76$, $P=0.007)$. While there was no association between either of these variants and HIV-negative TB, the same genotype trends were observed, suggesting that these variants may have a subtle influence on TB susceptibility, the impact of which is more pronounced in individuals already immunocompromised by HIV infection.

There was borderline evidence that the SLC11A1 exon 2 deletion may be associated with TB susceptibility in HIVpositive cases $(\mathrm{OR}=2.60,95 \% \mathrm{CI}=0.97-6.99, P=0.059)$. Heterozygosity for the SLC11A1 CAAA polymorphism was associated with protection in both HIV-positive (OR $=0.70$, 95\% CI $=0.49-0.99, P=0.046)$ and HIV-negative (OR $=$ $0.65,95 \% \mathrm{CI}=0.46-0.92, P=0.014) \mathrm{TB}$ cases.

\section{DISCUSSION}

In the Karonga population, heterozygosity for the $3^{\prime}$ untranslated (UTR) region CAAA insertion/deletion in SCL11A1 is associated with protection against TB, regardless of HIV status. This variant has apparently not been previously investigated for association with TB susceptibility. Furthermore, an association between any SCL11A1 variant and TB susceptibility in HIV-positive individuals has not been described previously. That this association is observed in both HIV-negative and HIV-positive cases suggests that the SCL11A1 gene product acts to protect against TB even during the immunosuppressed state of HIV infection, which may provide insight into how SCL11A11 influences TB susceptibility. The gene product is thought to act as a divalent ion pump that can influence acidity and/or iron content of the phagosome, and thus be involved in the direct killing of mycobacteria. However, it is also involved in regulation of the development of the adaptive immune response. As the adaptive immune response becomes impaired by infection and destruction of T cells in the course of HIV infection, these findings suggest SCL11A1 may be more important in the innate immune response to TB. It is interesting to contrast this with the growing evidence that suggests that SCL11A1 may influence development of the acquired immune response to leprosy. $^{23}$

It is not known whether the CAAA variant has any effect on the function or expression of the SCL11A1 gene product. Other SCL11A1 variants that have been associated with TB susceptibility elsewhere (including the promoter microsatellite, which does have a putative effect on the levels of SCL11A1 expression) were not associated in this Malawi series. The association of several variants of this gene with mycobacterial disease is unlikely to be due to chance, but the failure to identify a consistently associated variant may indicate that other, as yet undescribed, polymorphisms in this gene are responsible for the observed associations. A preliminary sequencing project of a 244-basepair segment of the $3^{\prime}$ UTR of the SLC11A1 gene in Malawians has not shown any novel polymorphisms. An alternative possibility is that the associations have arisen due to linkage disequilibrium with a variant in some neighboring gene that influences TB susceptibility.

There is some evidence that SCL11A1 genotype may influence susceptibility to HIV infection in Colombians. ${ }^{24}$ While preliminary results suggest that this is not the case in Karonga district, the possibility that the association observed in HIVpositive cases is due to susceptibility to HIV infection rather that TB infection cannot be ruled out.

On phagocytic cells complement receptor 1 mediates the adherence and phagocytosis of complement-opsonized pathogens. Since mycobacteria use this mechanism to gain entry 
TABle 2

Genotype frequencies for cases and controls and odds ratios (ORs)*

\begin{tabular}{|c|c|c|c|c|c|c|c|c|c|c|}
\hline \multirow[b]{2}{*}{ Gene } & \multirow[b]{2}{*}{ Polymorphism } & \multirow[b]{2}{*}{ Genotype } & \multicolumn{2}{|c|}{$\mathrm{HIV}+$ cases } & \multicolumn{2}{|c|}{ HIV- cases } & \multicolumn{2}{|c|}{ HIV- controls } & \multirow{2}{*}{$\begin{array}{l}\text { HIV- cases compared } \\
\text { to HIV-controls: } \\
\text { OR (95\% confidence } \\
\text { limits) } P \text { values }\end{array}$} & \multirow{2}{*}{$\begin{array}{l}\text { HIV+ cases compared } \\
\text { to HIV-controls: } \\
\text { OR (95\% confidence } \\
\text { limits) } P \text { values }\end{array}$} \\
\hline & & & $\%$ & $\mathrm{n}$ & $\%$ & $\mathrm{n}$ & $\%$ & $\mathrm{n}$ & & \\
\hline \multirow[t]{5}{*}{ CCL3 (MIPI- $\alpha)$} & Promoter microsatellite & $331 / 331$ & 48 & 99 & 48 & 70 & 39 & 226 & & \\
\hline & $(\mathrm{TA})_{\mathrm{n}}$ at -906 & $331 / 327$ & 24 & 50 & 27 & 39 & 28 & 162 & $0.69(0.40-1.16) 0.156$ & $0.74(0.46-1.18) 0.204$ \\
\hline & & $331 / 333$ & 18 & 37 & 15 & 22 & 19 & 113 & $0.55(0.28-1.03) 0.062$ & $0.65(0.38-1.09) 0.104$ \\
\hline & & Other & 10 & 20 & 11 & 16 & 14 & 79 & $0.65(0.32-1.33) 0.242$ & $0.67(0.35-1.28) 0.224$ \\
\hline & & & & 206 & & 147 & & 580 & & \\
\hline \multirow[t]{28}{*}{ CR1 } & $\mathrm{A} \rightarrow \mathrm{G}$ at 4795 & $\mathrm{a} / \mathrm{a}$ & 64 & 161 & 61 & 119 & 62 & 459 & & \\
\hline & K1590E & $\mathrm{a} / \mathrm{g}$ & 30 & 75 & 32 & 63 & 33 & 239 & $0.96(0.65-1.42) 0.844$ & $0.81(0.56-1.17) 0.255$ \\
\hline & $\left(\mathrm{McC}^{\mathrm{a}} \rightarrow \mathrm{McC}^{\mathrm{b}}\right)$ & $\mathrm{g} / \mathrm{g}$ & 6 & 16 & 7 & 13 & 5 & 36 & $1.75(0.82-3.74) 0.147$ & $1.23(0.59-2.56) 0.588$ \\
\hline & & & & 252 & & 195 & & 734 & & \\
\hline & $\mathrm{G} \rightarrow \mathrm{A}$ at 4828 & $\mathrm{~g} / \mathrm{g}$ & 46 & 115 & 50 & 97 & 48 & 350 & & \\
\hline & R1601G & $\mathrm{g} / \mathrm{a}$ & 40 & 101 & 38 & 73 & 42 & 306 & $0.91(0.62-1.32) 0.609$ & $0.96(0.67-1.38) 0.829$ \\
\hline & $(\mathrm{Sl1} \rightarrow \mathrm{Sl2})$ & $\mathrm{a} / \mathrm{a}$ & 14 & 35 & 12 & 24 & 10 & 72 & $1.48(0.82-2.66) 0.192$ & $1.33(0.77-2.32) 0.309$ \\
\hline & & & & 251 & & 194 & & 728 & & \\
\hline & $\mathrm{A} \rightarrow \mathrm{G}$ at 4870 & $\mathrm{~g} / \mathrm{g}$ & 80 & 201 & 78 & 151 & 79 & 578 & & \\
\hline & I1615V & $\mathrm{g} / \mathrm{a}$ & 18 & 46 & 21 & 41 & 19 & 138 & $1.30(0.84-2.01) 0.232$ & $0.90(0.58-1.41) 0.655$ \\
\hline & & $\mathrm{a} / \mathrm{a}$ & 1 & 3 & 1 & 2 & 2 & 11 & $1.04(0.20-5.23) 0.965$ & $0.53(0.10-2.96) 0.470$ \\
\hline & & & & 250 & & 194 & & 727 & & \\
\hline & $A \rightarrow G$ at 3650 & $\mathrm{a} / \mathrm{a}$ & 63 & 32 & 59 & 48 & 60 & 107 & & \\
\hline & H1208R & $\mathrm{a} / \mathrm{g}$ & 33 & 17 & 37 & 30 & 33 & 58 & $1.12(0.55-2.28) 0.759$ & $0.55(0.18-1.65) 0.287$ \\
\hline & & $\mathrm{g} / \mathrm{g}$ & 4 & 2 & 5 & 4 & 7 & 12 & $1.20(0.27-5.44) 0.809$ & $0.86(0.08-9.30) 0.904$ \\
\hline & & & & 51 & & 82 & & 177 & & \\
\hline & $\mathrm{T} \rightarrow \mathrm{C}$ at 2078 & $t / t$ & 77 & 95 & 79 & 96 & 76 & 262 & & \\
\hline & I684T & $t / c$ & 13 & 16 & 13 & 16 & 15 & 53 & $0.53(0.22-1.27) 0.155$ & $0.88(0.36-2.15) 0.778$ \\
\hline & & $\mathrm{c} / \mathrm{c}$ & 10 & 13 & 7 & 9 & 9 & 30 & $0.79(0.29-2.15) 0.644$ & $0.86(0.35-2.13) 0.747$ \\
\hline & & & & 124 & & 121 & & 345 & & \\
\hline & & $\mathrm{g} / \mathrm{g}$ & 71 & 164 & 68 & 133 & 74 & 496 & & \\
\hline & $\mathrm{G} \rightarrow \mathrm{T}$ at 3093 & $\mathrm{~g} / \mathrm{t}$ & 27 & 62 & 28 & 55 & 24 & 160 & $1.45(0.97-2.16) 0.073$ & $1.25(0.83-1.87) 0.290$ \\
\hline & Q1022H & $t / t$ & 2 & 4 & 4 & 8 & 2 & 14 & $3.12(1.13-8.60) 0.028$ & $0.99(0.26-3.76) 0.987$ \\
\hline & & & & 230 & & 196 & & 670 & & \\
\hline & & $\mathrm{c} / \mathrm{c}$ & 73 & 186 & 72 & 151 & 74 & 541 & & \\
\hline & $\mathrm{C} \rightarrow \mathrm{G}$ at 5507 & $\mathrm{c} / \mathrm{g}$ & 24 & 62 & 25 & 53 & 24 & 177 & $1.14(0.76-1.68) 0.529$ & $1.14(0.77-1.69) 0.515$ \\
\hline & P1827R & $\mathrm{g} / \mathrm{g}$ & 2 & 6 & 3 & 7 & 2 & 14 & $2.32(0.84-6.37) 0.104$ & $1.29(0.42-3.94) 0.652$ \\
\hline & & & & 254 & & 211 & & 732 & & \\
\hline ICAM1 & $\mathrm{A} \rightarrow \mathrm{T}$ at $179 \mathrm{~K} 29 \mathrm{M}$ & $\mathrm{a} / \mathrm{a}$ & 41 & 89 & 45 & 94 & 45 & 268 & & \\
\hline & $\left(\right.$ ICAM- $1^{\mathrm{ref}} \rightarrow$ & $\mathrm{a} / \mathrm{t}$ & 40 & 86 & 37 & 78 & 37 & 222 & $0.96(0.65-1.42) 0.852$ & $0.97(0.62-1.51) 0.890$ \\
\hline & ICAM-1 $\left.{ }^{\text {Kilifi }}\right)$ & $t / t$ & 19 & 42 & 18 & 37 & 18 & 106 & $0.97(0.60-1.57) 0.915$ & $0.94(0.55-1.62) 0.827$ \\
\hline & & & & 217 & & 209 & & 596 & & \\
\hline IFNG & Intron $1+874$ & $\mathrm{a} / \mathrm{a}$ & 65 & 154 & 71 & 151 & 66 & 467 & & \\
\hline & $\mathrm{T} \rightarrow \mathrm{A}$ & $\mathrm{a} / \mathrm{t}$ & 31 & 74 & 28 & 60 & 31 & 219 & $0.92(0.63-1.32) 0.617$ & $0.96(0.66-1.43) 0.885$ \\
\hline & & $t / t$ & 4 & 10 & 1 & 2 & 2 & 17 & $0.40(0.08-1.84) 0.233$ & $0.67(0.58-4.06) 0.386$ \\
\hline & & & & 238 & & 213 & & 703 & & \\
\hline IL10 & -592 & $\mathrm{c} / \mathrm{c}$ & 38 & 94 & 40 & 85 & 42 & 297 & & \\
\hline & & $\mathrm{c} / \mathrm{a}$ & 49 & 120 & 47 & 98 & 43 & 301 & $1.02(0.70-1.49) 0.905$ & $1.23(0.85-1.80) 0.274$ \\
\hline & & $\mathrm{a} / \mathrm{a}$ & 13 & 32 & 13 & 27 & 15 & 107 & $0.91(0.53-1.57) 0.745$ & $0.92(0.54-1.57) 0.758$ \\
\hline & & & & 246 & & 210 & & 705 & & \\
\hline & -819 & $\mathrm{c} / \mathrm{c}$ & 38 & 93 & 40 & 85 & 41 & 287 & & \\
\hline & & $\mathrm{c} / \mathrm{t}$ & 49 & 122 & 47 & 98 & 43 & 303 & $0.98(0.68-1.43) 0.922$ & $1.13(0.78-1.65) 0.513$ \\
\hline & & $t / t$ & 13 & 32 & 13 & 27 & 15 & 108 & $0.87(0.51-1.51) 0.628$ & $0.86(0.51-1.48) 0.593$ \\
\hline & & & & 247 & & 210 & & 698 & & \\
\hline & -1082 & $\mathrm{a} / \mathrm{a}$ & 47 & 73 & 41 & 69 & 38 & 203 & & \\
\hline & & $\mathrm{a} / \mathrm{g}$ & 42 & 65 & 46 & 78 & 46 & 251 & $1.16(0.76-1.76) 0.497$ & $0.55(0.34-0.88) 0.013$ \\
\hline & & $\mathrm{g} / \mathrm{g}$ & 11 & 17 & 13 & 23 & 16 & 87 & $0.83(0.46-1.50) 0.538$ & $0.37(0.18-0.76) 0.007$ \\
\hline & & & & 155 & & 170 & & 541 & & \\
\hline LTA (TNF $\beta)$ & 5'UTR microsatellite & 101 absent & 89 & 211 & 87 & 172 & 85 & 599 & & \\
\hline & $(\mathrm{AC} / \mathrm{GT})_{\mathrm{n}}$ at $-3.5 \mathrm{~kb} \dagger$ & 1011 сору & 10 & 24 & 13 & 26 & 15 & 105 & $0.89(0.52-1.52) 0.658$ & $0.64(0.38-1.07) 0.090$ \\
\hline & & 1012 copies & 1 & 2 & 0 & 0 & 0.4 & 3 & & \\
\hline & & & & 237 & & 198 & & 707 & & \\
\hline & & 103 absent & 66 & 156 & 62 & 122 & 62 & 436 & & \\
\hline & & 1031 сору & 30 & 71 & 34 & 67 & 34 & 241 & $1.04(0.74-1.47) 0.827$ & $0.78(0.55-1.11) 0.163$ \\
\hline & & 1032 copies & 4 & 10 & 4 & 9 & 4 & 30 & & \\
\hline & & & & 237 & & 198 & & 707 & & \\
\hline & & 105 absent & 47 & 111 & 52 & 102 & 54 & 380 & & \\
\hline & & 1051 сору & 43 & 101 & 40 & 80 & 39 & 274 & $1.06(0.77-1.45) 0.728$ & $1.12(0.83-1.51) 0.444$ \\
\hline & & 1052 copies & 10 & 25 & 8 & 16 & 7 & 53 & & \\
\hline & & & & 237 & & 198 & & 707 & & \\
\hline
\end{tabular}


TABLE 2

Continued

\begin{tabular}{|c|c|c|c|c|c|c|c|c|c|c|}
\hline \multirow[b]{2}{*}{ Gene } & \multirow[b]{2}{*}{ Polymorphism } & \multirow[b]{2}{*}{ Genotype } & \multicolumn{2}{|c|}{$\mathrm{HIV}+$ cases } & \multicolumn{2}{|c|}{ HIV- cases } & \multicolumn{2}{|c|}{ HIV- controls } & \multirow{2}{*}{$\begin{array}{l}\text { HIV- cases compared } \\
\text { to HIV-controls: } \\
\text { OR (95\% confidence } \\
\text { limits) } P \text { values }\end{array}$} & \multirow{2}{*}{$\begin{array}{l}\text { HIV+ cases compared } \\
\text { to HIV-controls: } \\
\text { OR (95\% confidence } \\
\text { limits) } P \text { values }\end{array}$} \\
\hline & & & $\%$ & $\mathrm{n}$ & $\%$ & $\mathrm{n}$ & $\%$ & $\mathrm{n}$ & & \\
\hline & & 111 absent & 94 & 223 & 95 & 188 & 94 & 668 & & \\
\hline & & 1111 copy & 6 & 14 & 5 & 10 & 6 & 39 & $1.26(0.55-2.85) 0.583$ & $0.86(0.41-1.78) 0.677$ \\
\hline & & 1112 copies & 0 & 0 & 0 & 0 & 0 & 0 & & \\
\hline & & & & 237 & & 198 & & 707 & & \\
\hline \multirow{4}{*}{$\begin{array}{l}\text { MBL2 (MBP, } \\
\text { MBL) }\end{array}$} & $\mathrm{G} \rightarrow \mathrm{A}$ at 239 & $g / g$ & 62 & 95 & 65 & 110 & 66 & 362 & & \\
\hline & G57E & $\mathrm{g} / \mathrm{a}$ & 34 & 53 & 31 & 52 & 29 & 160 & $1.16(0.74-1.80) 0.522$ & $1.69(1.04-2.75) 0.034$ \\
\hline & (C variant) & $\mathrm{a} / \mathrm{a}$ & 4 & 6 & 4 & 6 & 5 & 24 & $0.89(0.31-2.52) 0.823$ & $1.07(0.33-3.50) 0.908$ \\
\hline & & & & 154 & & 168 & & 546 & & \\
\hline SLC11A1 & Promoter & $199 / 199$ & 62 & 163 & 62 & 145 & 60 & 469 & & \\
\hline \multirow[t]{15}{*}{ (NRAMP1) } & microsatellite (GT)n & 199/201 & 30 & 79 & 31 & 71 & 34 & 266 & $0.88(0.62-1.25) 0.481$ & $0.75(0.52-1.08) 0.123$ \\
\hline & $(199=$ allele 3 & 201/201 & 6 & 15 & 3 & 7 & 4 & 32 & $0.71(0.28-1.78) 0.463$ & $1.11(0.51-2.40) 0.796$ \\
\hline & $201=$ allele 2$)$ & Other & 2 & 4 & 4 & 9 & 1 & 11 & & \\
\hline & & & & 261 & & 232 & & 778 & & \\
\hline & TGTG ins/del & ins/ins & 58 & 126 & 51 & 112 & 50 & 353 & & \\
\hline & & ins/del & 34 & 74 & 42 & 91 & 40 & 287 & $1.01(0.71-1.42) 0.970$ & $0.72(0.49-1.07) 0.108$ \\
\hline & & del/del & 8 & 17 & 7 & 15 & 10 & 69 & $0.68(0.34-1.37) 0.282$ & $0.62(0.32-1.18) 0.147$ \\
\hline & & & & 217 & & 218 & & 709 & & \\
\hline & CAAA ins/del & del/del & 50 & 129 & 49 & 117 & 42 & 317 & & \\
\hline & & ins/del & 40 & 103 & 38 & 90 & 48 & 367 & $0.65(0.46-0.92) 0.014$ & $0.70(0.49-0.99) 0.046$ \\
\hline & & ins/ins & 10 & 27 & 13 & 32 & 10 & 78 & $1.11(0.64-1.91) 0.719$ & $1.01(0.57-1.79) 0.971$ \\
\hline & & & & 259 & & 239 & & 762 & & \\
\hline & Exon 29 bp del & ins/ins & 93 & 152 & 93 & 155 & 94 & 493 & & \\
\hline & & Deletion & & & & & & & & \\
\hline & & present & 7 & $\begin{array}{r}11 \\
163\end{array}$ & 7 & $\begin{array}{r}11 \\
166\end{array}$ & 6 & $\begin{array}{r}32 \\
525\end{array}$ & $0.88(0.38-2.02) 0.757$ & $2.60(0.97-6.99) 0.059$ \\
\hline \multirow[t]{16}{*}{ TLR2 } & Intron 2 microsat $\neq$ & 216 absent & 86 & 215 & 89 & 192 & 89 & 658 & & \\
\hline & & 2161 copy & 14 & 34 & 10 & 21 & 11 & 83 & $1.29(0.72-2.32) 0.393$ & $1.40(0.84-2.36) 0.201$ \\
\hline & & 2162 copies & 0 & 0 & 1 & 2 & 0.1 & 1 & & \\
\hline & & & & 249 & & 215 & & 742 & & \\
\hline & & 222 absent & 77 & 191 & 75 & 162 & 74 & 550 & & \\
\hline & & 2221 copy & 21 & 52 & 23 & 49 & 25 & 182 & $0.94(0.63-1.40) 0.763$ & $1.04(0.70-1.53) 0.863$ \\
\hline & & 2222 copies & 2 & 6 & 2 & 4 & 1 & 10 & & \\
\hline & & & & 249 & & 215 & & 742 & & \\
\hline & & 224 absent & 43 & 106 & 42 & 90 & 41 & 304 & & \\
\hline & & 2241 copy & 43 & 107 & 43 & 93 & 48 & 358 & $1.02(0.76-1.37) 0.908$ & $1.19(0.89-1.59) 0.251$ \\
\hline & & 2242 copies & 14 & 36 & 15 & 32 & 11 & 80 & & \\
\hline & & & & 249 & & 215 & & 742 & & \\
\hline & & 226 absent & 79 & 196 & 76 & 164 & 77 & 569 & & \\
\hline & & 2261 copy & 18 & 46 & 23 & 49 & 22 & 166 & $0.96(0.64-1.44) 0.841$ & $1.15(0.76-1.72) 0.507$ \\
\hline & & 2262 copies & 3 & 7 & 1 & 2 & 1 & 7 & & \\
\hline & & & & 249 & & 215 & & 742 & & \\
\hline TLR4 & Exon 4 & $\mathrm{a} / \mathrm{a}$ & 93 & 112 & 90 & 146 & 91 & 389 & & \\
\hline & $\mathrm{A} \rightarrow \mathrm{G}$ at 896 & $\mathrm{~g} / \mathrm{a}$ & 7 & 8 & 10 & 16 & 9 & 38 & $1.16(0.58-2.31) 0.677$ & $0.98(0.32-3.02) 0.976$ \\
\hline & D299G & $\mathrm{g} / \mathrm{g}$ & 0 & 0 & 0 & 0 & 0 & 0 & & \\
\hline & & & & 120 & & 162 & & 427 & & \\
\hline TNF & -238 & $\mathrm{~g} / \mathrm{g}$ & 85 & 122 & 90 & 162 & 87 & 464 & & \\
\hline & & $\mathrm{g} / \mathrm{a}$ & 15 & 21 & 10 & 19 & 13 & 69 & $0.82(0.45-1.49) 0.515$ & $1.37(0.68-2.73) 0.378$ \\
\hline & & $\mathrm{a} / \mathrm{a}$ & 0.7 & 1 & 0 & 0 & 0 & 0 & & \\
\hline & & & & 144 & & 181 & & 533 & & \\
\hline & -308 & $g / g$ & 82 & 97 & 82 & 132 & 83 & 344 & & \\
\hline & & $\mathrm{g} / \mathrm{a}$ & 18 & 21 & 17 & 27 & 17 & 71 & 1.15 (0.67-1.99) 0.610 & $1.31(0.59-2.90) 0.511$ \\
\hline & & $\mathrm{a} / \mathrm{a}$ & 0 & 0 & 1 & 2 & 0.2 & 1 & $1.65(0.10-27.78) 0.730$ & \\
\hline & & & & 118 & & 161 & & 416 & & \\
\hline & -863 & $\mathrm{c} / \mathrm{c}$ & 82 & 98 & 78 & 129 & 72 & 301 & & \\
\hline & & $a / c$ & 17 & 20 & 20 & 33 & 26 & 109 & $0.73(0.45-1.19) 0.204$ & $0.59(0.29-1.20) 0.145$ \\
\hline & & $\mathrm{a} / \mathrm{a}$ & 1 & 1 & 2 & 3 & 2 & 7 & $2.07(0.37-11.47) 0.407$ & $0.71(0.03-15.69) 0.829$ \\
\hline & & & & 119 & & 165 & & 417 & & \\
\hline VDR & $\mathrm{T} \rightarrow \mathrm{C} 352$ silent & $t / t$ & 59 & 132 & 61 & 129 & 57 & 384 & & \\
\hline & $(\operatorname{Taq} \mathrm{I}, \mathrm{T} \rightarrow \mathrm{t})$ & $t / c$ & 36 & 82 & 34 & 72 & 36 & 241 & $0.82(0.56-1.19) 0.285$ & $0.94(0.64-1.38) 0.747$ \\
\hline & & $\mathrm{c} / \mathrm{c}$ & 5 & 11 & 5 & 11 & 7 & 47 & $0.59(0.29-1.23) 0.162$ & $0.80(0.36-1.78) 0.583$ \\
\hline & & & & 225 & & 212 & & 672 & & \\
\hline & $\mathrm{G} \rightarrow \mathrm{T}$ & $t / t$ & 49 & 97 & 48 & 70 & 53 & 287 & & \\
\hline & $(A p a \mathrm{I}, \mathrm{a} \rightarrow \mathrm{A})$ & $t / g$ & 41 & 82 & 43 & 63 & 39 & 210 & $1.36(0.88-2.11) 0.169$ & $1.23(0.81-1.87) 0.322$ \\
\hline & & $\mathrm{g} / \mathrm{g}$ & 10 & 20 & 9 & 13 & 8 & 46 & $0.98(0.44-2.20) 0.971$ & $1.10(0.56-2.18) 0.783$ \\
\hline & & & & 199 & & 146 & & 543 & & \\
\hline
\end{tabular}


TABLE 2

Continued

\begin{tabular}{|c|c|c|c|c|c|c|c|c|c|c|}
\hline \multirow[b]{2}{*}{ Gene } & \multirow[b]{2}{*}{ Polymorphism } & \multirow[b]{2}{*}{ Genotype } & \multicolumn{2}{|c|}{$\mathrm{HIV}+$ cases } & \multicolumn{2}{|c|}{ HIV- cases } & \multicolumn{2}{|c|}{ HIV- controls } & \multirow{2}{*}{$\begin{array}{l}\text { HIV- cases compared } \\
\text { to HIV-controls: } \\
\text { OR ( } 95 \% \text { confidence } \\
\text { limits) } P \text { values }\end{array}$} & \multirow{2}{*}{$\begin{array}{l}\text { HIV+ cases compared } \\
\text { to HIV-controls: } \\
\text { OR ( } 95 \% \text { confidence } \\
\text { limits) } P \text { values }\end{array}$} \\
\hline & & & $\%$ & $\mathrm{n}$ & $\%$ & $\mathrm{n}$ & $\%$ & $\mathrm{n}$ & & \\
\hline & $\mathrm{C} \rightarrow \mathrm{T}$ & $\mathrm{c} / \mathrm{c}$ & 62 & 123 & 61 & 89 & 58 & 314 & & \\
\hline & \multirow{3}{*}{$(B s m \mathrm{I}, \mathrm{b} \rightarrow \mathrm{B})$} & $\mathrm{c} / \mathrm{t}$ & 35 & 69 & 37 & 54 & 35 & 192 & $1.05(0.67-1.65) 0.824$ & $0.85(0.56-1.28) 0.432$ \\
\hline & & $t / t$ & 3 & 6 & 3 & 4 & 7 & 39 & $0.70(0.22-2.25) 0.546$ & $0.64(0.23-1.77) 0.386$ \\
\hline & & & & 198 & & 147 & & 545 & & \\
\hline
\end{tabular}

Statistically significant or marginally significant associations are shown in bold. HIV = human immunodeficiency virus; CCL3 = chemokine (C-C motif) ligand 3; CR1 = complement receptor 1 ; ICAM1 $=$ intercellular adhesion molecule 1 ; IFNG $=$ interferon- $\gamma$; IL10 = interleukin 10; LTA = lymphotoxin alpha; TNF $\beta=$ tumor necrosis factor $\beta$; UTR $=$ untranslated region; $\mathrm{MBL}=$ mannose-binding lectin; SLC11A1 = solute carrier family 11, member 1; ins = insertion; del = deletion; TLR2 = toll-like receptor 2; TNF = tumor necrosis factor; VDR = vitamin D receptor.

$\dagger$ Baseline alleles other than 101, 103, 105, and 111.

$\ddagger$ Baseline alleles other than 216, 222, 224, 226 .

into macrophages, it was hypothesized that variants in CR1 may influence susceptibility to TB. ${ }^{17,25,26}$ Five polymorphisms that encode amino acid substitutions in the extra-membrane portion of the protein and which may affect either ligand binding or CR1 stability were investigated. ${ }^{17}$ Homozygosity for $\mathrm{Q} 1022 \mathrm{H}$ was associated with $\mathrm{TB}$ susceptibility $(\mathrm{OR}=$ $3.12,95 \% \mathrm{CI}=1.13-8.60, P=0.028)$. This variant occurs in a ligand binding domain, close to an aspartic acid residue that is important in $\mathrm{C} 3 \mathrm{~b}$ binding. It has been suggested that substitution of glutamine to a weakly basic and more hydrophobic histidine residue may alter ligand binding by CR1. ${ }^{27}$ If this variant does reduce $\mathrm{CR} 1$ binding of $\mathrm{C} 3 \mathrm{~b}$, it appears that rather than providing protection by limiting $M$. tuberculosis uptake, it increases TB susceptibility, indicating that uptake via CR1 may invoke protective mechanisms. Lack of an association between Q1022H and TB in HIV-positive individuals suggests that this protective mechanism is inhibited or ablated in immunosuppressed individuals.

Interferon- $\gamma$ is an essential and central regulator of the human response to infection. Homozygosity for the $\mathrm{T}$ allele of an intronic single nucleotide polymorphism (SNP) at +874 that may influence IFN- $\gamma$ production is associated with susceptibility to TB in Sicilians and in South Africans. ${ }^{14-16}$ This SNP was not associated with TB susceptibility in Malawi. This could be because the lower allele frequency in Malawi $(20 \%$ compared with $50 \%$ ) reduces the power to detect such an association, or because the TB association is due to another polymorphism that is in linkage disequilibrium (LD) with the +874 SNP in Sicily but not in Malawi, or because this allele does not affect TB susceptibility in this population. In some populations it appears that the +874 SNP is in complete LD with a microsatellite allele that is associated with increased IFN- $\gamma$ production. Genotyping of this microsatellite indicates that while there is evidence of strong LD between it and the +874 SNP in Malawi, this is not absolute.

The gene product of MBL2, mannose-binding lectin, binds to mannose groups on a variety of bacteria, resulting in complement activation and opsonophagocytosis. Three polymorphisms in exon 1 influence serum MBL levels. Low serum concentrations of MBL may be associated with recurrent infections in young children, and yet low producing alleles are maintained in all populations, leading to the hypothesis that these variants are maintained by balancing selection. ${ }^{17}$ Protection from mycobacterial infection by limiting phagocytotic entry of mycobacteria into host cells has been proposed as a mechanism by which such alleles may be maintained. ${ }^{17}$ There is some evidence that such variants may be protective against meningeal TB in Cape Coloureds but no association with protection against pulmonary $\mathrm{TB}$ in The Gambia was found. ${ }^{13,28}$ In Malawi, we found no convincing evidence of association with TB.

Interleukin-10 is a key anti-inflammatory cytokine. Three promoter polymorphisms $(-1082,-819,-592)$ that may influence IL10 levels were investigated for susceptibility to TB. ${ }^{17}$ The HIV-positive, but not HIV-negative, Malawians homozygous for the $-1082 \mathrm{G}$ allele appeared to be protected against TB. The -1082 SNP lies in a putative transcription factor binding site, but reports on the function of the IL10 promoter variants are contradictory. ${ }^{29}$ The $-1082 \mathrm{G}$ allele has been reported to be associated with reduced IL10 transcription, but haplotypes bearing the $G$ variant are usually associated with increased IL10 levels. ${ }^{30,31}$ It is unclear how increased IL10 production would provide protection against TB. However, there is some evidence that other, more distal IL10 promoter polymorphisms may be more important in controlling IL10 levels than the proximal polymorphisms investigated here. ${ }^{32}$ Furthermore, one haplotype of these distal polymorphisms is reported to be associated with increased IL10 production and is linked to each of the three common proximal haplotypes, perhaps explaining the variable results of studies of the proximal polymorphisms. In light of the observed $-1082 \mathrm{G}$ association, an investigation of these distal polymorphisms and the haplotypes that are present in the Malawi population is warranted.

Intracellular adhesion molecule 1 (ICAM1/CD54) mediates both leukocyte-leukocyte and leukocyte-endothelial interactions during an immune response. ${ }^{33} \mathrm{An}$ apparently deleterious polymorphism, K29M, which reduces binding to leukocyte function associated antigen-1 and fibrinogen, two of the key ligands for ICAM1, has been postulated to be maintained by balancing selection. However, there was no evidence to suggest that this polymorphism influences TB susceptibility in Malawi. ${ }^{33}$

Vitamin D receptor is the receptor for the active form of vitamin $\mathrm{D}\left(1 \alpha 25(\mathrm{OH})_{2} \mathrm{D} 3\right)$, which in addition to influencing bone mass, has important immuno-modulatory effects including suppression of cytokine synthesis, immunoglobulin production and lymphocyte proliferation. Epidemiologic evidence suggests vitamin $\mathrm{D}$ deficiency is associated with susceptibility to TB. There is evidence that vitamin D impedes growth of $M$. tuberculosis in human macrophage and monocytic cell lines, and it has been suggested that vitamin $D$ containing medications may be useful in treating some forms of leprosy. ${ }^{17,34}$ Homozygosity for a Taq 1 polymorphism (tt) 
in the ligand binding domain of VDR was associated with resistance to TB in The Gambia. ${ }^{11}$ Although this genotype was associated with susceptibility to leprosy, there was no evidence that it influences TB susceptibility in Malawians. ${ }^{17}$ Failure to replicate the association observed in The Gambia may indicate that VDR does not influence TB susceptibility in Malawi, but it is also possible that the Gambian association was due to linkage disequilibrium between the silent Taq 1 SNP in codon 352 and another polymorphism with a functional effect, which does not exhibit the same LD in the Malawi population. Among HIV infected Malawians (Table 1) homozygosity for the intronic VDR polymorphism Bsm I (BB) appeared to be protective against $\mathrm{TB}$, but this mainly reflects a high incidence of this genotype in the small sample of HIV-positive controls. Interestingly, the same BB genotype has been associated with rapid HIV disease progression in Spaniards, but association between VDR and HIV susceptibility was not assessed in that study. ${ }^{35}$

On recognition of pathogens, mammalian toll-like receptors send signals to activate innate and adaptive immunity. Both TLR2 and TLR4 can mediate cellular activation in response to components of M. tuberculosis. ${ }^{36}$ An intronic microsatellite that may influence TLR2 expression levels has been identified in intron 2 of the TLR2 gene but no alleles were associated with TB susceptibility in Malawi (Segal S and others, unpublished). A TLR4 polymorphism that results in an amino acid substitution (Arg299Gly) in the receptor's extra-cellular domain, and thus impairs lipolysaccharideinduced signaling, was found not to associate with TB susceptibility in Malawi. ${ }^{17}$

Tumor necrosis factor is a proinflammatory cytokine with pleiotrophic effects. Its critical role in protection against TB is illustrated by the observation that some patients with inflammatory conditions that have been treated with the anti-TNF drug infliximab develop active TB. ${ }^{37}$ Several promoter polymorphisms exist that may influence TNF expression and some have been associated with both autoimmune and infectious diseases, although none have been associated with TB susceptibility. ${ }^{17,38-40}$ Consistent with these findings, no TNF promoter polymorphisms were associated with TB susceptibility in Malawi.

Some limited evidence that an LTA microsatellite allele may be protective against TB in HIV-positive Malawians was found. Like TNF, LTA is located in the HLA class III region, and encodes a cytokine with pleiotrophic immunomodulatory effects. Lymphotoxin alpha appears to be crucial in organizing the granulomatous response that is required for controlling M. tuberculosis infection in mice. ${ }^{41}$ Recently, two LTA SNPs were shown to be associated with susceptibility to myocardial infarction, and, these SNPs appear to influence the expression and/or function of LTA. ${ }^{42}$ It would be of interest to investigate the relationship between the upstream microsatellite and these putative functional polymorphisms. Any investigation of association with TNF and LTA is complicated by the fact that these genes lie in the HLA region and there may be linkage disequilibrium between HLA alleles that influence TB susceptibility. There was some evidence that the TNF-238a allele may be associated with HIV infection in Malawi. Variants in TNF have been associated with HIV progression in some populations, but not with susceptibility. ${ }^{43}$

The chemokine (C-C motif) ligand 3, formerly known as the macrophage inflammatory protein-1, induces activation of macrophages and proliferation and TH1 differentiation of $\mathrm{T}$ cells. ${ }^{44}$ It belongs to the $\mathrm{C}-\mathrm{C}$ chemokine family and binds receptors CCR-1, CCR-4, and CCR-5. Expression of CCL3 is induced by $M$. tuberculosis infection of human alveolar macrophages, with avirulent strains inducing greater expression than virulent strains. ${ }^{45}$ There was borderline evidence that the promoter microsatellite 333 allele might be associated with protection against TB in HIV-negative Malawians. However, the functional effect, if any, of this promoter microsatellite is unknown.

This large-scale investigation of candidate genes has shown that genetic variants in SCLA11A1 appear to influence TB susceptibility in Malawi, as has been found in other populations. Furthermore, this influence may extend to HIVinfected individuals. However, the association in Malawi is with a different variant of SCLA11A1 from that reported in other populations, and the molecular basis of this interpopulation heterogeneity requires further analysis. Previously reported associations between variants of VDR, MBL, and IFNG and TB susceptibility in HIV-negative individuals were not replicated in this Malawian population. An association with a novel candidate gene, CR1, was shown. This is the first study to report analysis of genetic variants that may influence TB susceptibility in individuals immunocompromised by HIV infection, and we found preliminary evidence that both an IL10 promoter variant and the Bsm I polymorphism in VDR may influence TB susceptibility in such individuals.

Overall, this extensive analysis highlights two important features of TB genetic susceptibility: the likely existence of important interpopulation differences in relevant susceptibility loci and the potential for identification of false-positive associations by analysis of variants in multiple genes. To address both issues, future studies of polygenic susceptibility will require larger sample sizes than generally studied to date, as well as replication studies, ideally in both the original and in different populations.

Received August 28, 2003. Accepted for publication March 11, 2004

Acknowledgments: We thank the many field, laboratory, and data management staff of the KPS who have carried out the work in Karonga District since 1979, the people of Karonga District, and the Ministry of Health and Population and the National Health Sciences Research Committee of Malawi for their encouragement of the KPS over many years. We also thank past and present Wellcome Trust Centre for Human Genetics (WTCHG) researchers and collaborators including Peter Zimmerman, Patricia Ramaley, and Graham Cooke for their role in developing the genotyping methods used here, as well as WTCHG core facilities staff, and Christophe Aucan, Kerrie Tosh, and Branwen Hennig for various assistance.

Financial support: This study was supported by the Wellcome Trust. Adrian V. S. Hill is a Wellcome Trust Principal Research Fellow.

Authors' addresses: Jodene Fitness, School of Biological Sciences, Victoria University of Wellington, PO Box 600, Wellington, New Zealand, E-mail: jodene.fitness@vuw.ac.nz. Sian Floyd and Paul E. M. Fine, Department of Tropical Hygiene, London School of Hygiene and Tropical Medicine, Keppel Street, London WC1E 7HT, United Kingdom, E-mails: sian.floyd@lshtm.ac.uk and paul.fine@1shtm.ac.uk. David K. Warndorff, Lifted Sichali, Simon Malema, and Amelia C. Crampin, Karonga Prevention Study, PO Box 46, Chilumba, Karonga District, Malawi. Adrian V. S. Hill, Wellcome Trust Centre for Human Genetics, Roosevelt Drive, Oxford OX3 7BN, United Kingdom, E-mail: adrian.hill@molecularmedicine.oxford.ac.uk. 


\section{REFERENCES}

1. Corbett EL, Watt CJ, Walker N, Maher D, Williams BG, Raviglione MC, Dye C, 2003. The growing burden of tuberculosis: global trends and interactions with the HIV epidemic. Arch Intern Med 163: 1009-1021.

2. Bloom BR, Small PM, 1998. The evolving relation between humans and Mycobacterium tuberculosis. N Engl J Med 338: 677678.

3. Bellamy R, 2003. Interferon-gamma and host susceptibility to tuberculosis. Am J Respir Crit Care Med 167: 946-947.

4. Marquet S, Schurr E, 2001. Genetics of susceptibility to infectious diseases: tuberculosis and leprosy as examples. Drug Metab Dispos 29: 479-483.

5. Shaw MA, Collins A, Peacock CS, Miller EN, Black GF, Sibthorpe D, Lins-Lainson Z, Shaw JJ, Ramos F, Silveira F, Blackwell JM, 1997. Evidence that genetic susceptibility to Mycobacterium tuberculosis in a Brazilian population is under oligogenic control: linkage study of the candidate genes NRAMP1 and TNFA. Tuber Lung Dis 78: 35-45.

6. Gao PS, Fujishima S, Mao XQ, Remus N, Kanda M, Enomoto T, Dake Y, Bottini N, Tabuchi M, Hasegawa N, Yamaguchi K, Tiemessen C, Hopkin JM, Shirakawa T, Kishi F, 2000. Genetic variants of NRAMP1 and active tuberculosis in Japanese populations. International Tuberculosis Genetics Team. Clin Genet 58: 74-76.

7. Bellamy R, Ruwende C, Corrah T, McAdam KP, Whittle HC, Hill AV, 1998. Variations in the NRAMP1 gene and susceptibility to tuberculosis in west Africans. N Engl J Med 338: 640-644.

8. Ryu S, Park YK, Bai GH, Kim SJ, Park SN, Kang S, 2000. 3'UTR polymorphisms in the NRAMP1 gene are associated with susceptibility to tuberculosis in Koreans. Int J Tuberc Lung Dis 4: 577-580.

9. Cervino AC, Lakiss S, Sow O, Hill AV, 2000. Allelic association between the NRAMP1 gene and susceptibility to tuberculosis in Guinea-Conakry. Ann Hum Genet 64: 507-512.

10. Greenwood CM, Fujiwara TM, Boothroyd LJ, Miller MA, Frappier D, Fanning EA, Schurr E, Morgan K, 2000. Linkage of tuberculosis to chromosome 2q35 loci, including NRAMP1, in a large aboriginal Canadian family. Am J Hum Genet 67: 405416.

11. Bellamy R, Ruwende C, Corrah T, McAdam KP, Thursz M, Whittle HC, Hill AV, 1999. Tuberculosis and chronic hepatitis $B$ virus infection in Africans and variation in the vitamin D receptor gene. J Infect Dis 179: 721-724.

12. Selvaraj P, Narayanan PR, Reetha AM, 1999. Association of functional mutant homozygotes of the mannose binding protein gene with susceptibility to pulmonary tuberculosis in India. Tuber Lung Dis 79: 221-227.

13. Hoal-Van Helden EG, Epstein J, Victor TC, Hon D, Lewis LA, Beyers N, Zurakowski D, Ezekowitz AB, van Helden PD, 1999. Mannose-binding protein B allele confers protection against tuberculous meningitis. Pediatr Res 45: 459-464.

14. Pravica V, Perrey C, Stevens A, Lee JH, Hutchinson IV, 2000. A single nucleotide polymorphism in the first intron of the human IFN-gamma gene: absolute correlation with a polymorphic CA microsatellite marker of high IFN-gamma production. Hum Immunol 61: 863-866.

15. Rossouw M, Nel HJ, Cooke GS, van Helden PD, Hoal EG, 2003. Association between tuberculosis and a polymorphic NFkappaB binding site in the interferon gamma gene. Lancet 361: 18711872.

16. Lio D, Marino V, Serauto A, Gioia V, Scola L, Crivello A, Forte GI, Colonna-Romano G, Candore G, Caruso C, 2002. Genotype frequencies of the $+874 \mathrm{~T} \rightarrow \mathrm{A}$ single nucleotide polymorphism in the first intron of the interferon-gamma gene in a sample of Sicilian patients affected by tuberculosis. Eur J Immunogenet 29: 371-374.

17. Fitness J, Floyd S, Warndorff DK, Sichali L, Mwaungulu L, Crampin AC, Fine PEM, Hill AVS, 2004. Large-scale candidate gene study of leprosy susceptibility in the Karonga District of northern Malawi. Am J Trop Med Hyg 71: 330-340.

18. Kang TJ, Chae GT, 2001. Detection of Toll-like receptor 2
(TLR2) mutation in the lepromatous leprosy patients. FEMS Immunol Med Microbiol 31: 53-58.

19. Glynn JR, Jenkins PA, Fine PE, Ponnighaus JM, Sterne JA, Mkandwire PK, Nyasulu S, Bliss L, Warndorff DK, 1995. Patterns of initial and acquired antituberculosis drug resistance in Karonga District, Malawi. Lancet 345: 907-910.

20. Ponninghaus JM, Fine PE, Bliss L, Sliney IJ, Bradley DJ, Rees RJ, 1987. The Lepra Evaluation Project (LEP), an epidemiological study of leprosy in Northern Malawi. I. Methods. Lepr Rev 58: 359-375.

21. Crampin AC, Mwinuka V, Malema SS, Glynn JR, Fine PE, 2001. Field-based random sampling without a sampling frame: control selection for a case-control study in rural Africa. Trans $R$ Soc Trop Med Hyg 95: 481-483.

22. Glynn JR, Warndorff DK, Malema SS, Mwinuka V, Ponnighaus JM, Crampin AC, Fine PE, 2000. Tuberculosis: associations with HIV and socioeconomic status in rural Malawi. Trans $R$ Soc Trop Med Hyg 94: 500-503.

23. Fitness J, Tosh K, Hill AV, 2002. Genetics of susceptibility to leprosy. Genes Immun 3: 441-453.

24. Marquet S, Sanchez FO, Arias M, Rodriguez J, Paris SC, Skamene E, Schurr E, Garcia LF, 1999. Variants of the human NRAMP1 gene and altered human immunodeficiency virus infection susceptibility. J Infect Dis 180: 1521-1525.

25. Schlesinger LS, Horwitz MA, 1990. Phagocytosis of leprosy bacilli is mediated by complement receptors CR1 and CR3 on human monocytes and complement component $\mathrm{C} 3$ in serum. J Clin Invest 85: 1304-1314.

26. Hirsch CS, Ellner JJ, Russell DG, Rich EA, 1994. Complement receptor-mediated uptake and tumor necrosis factor-alphamediated growth inhibition of Mycobacterium tuberculosis by human alveolar macrophages. J Immunol 152: 743-753.

27. Xiang L, Rundles JR, Hamilton DR, Wilson JG, 1999. Quantitative alleles of CR1: coding sequence analysis and comparison of haplotypes in two ethnic groups. J Immunol 163: 4939-4945.

28. Bellamy R, Ruwende C, McAdam KP, Thursz M, Sumiya M, Summerfield J, Gilbert SC, Corrah T, Kwiatkowski D, Whittle HC, Hill AV, 1998. Mannose binding protein deficiency is not associated with malaria, hepatitis B carriage nor tuberculosis in Africans. QJM 91: 13-18.

29. Kube D, Platzer C, von Knethen A, Straub H, Bohlen H, Hafner $\mathrm{M}$, Tesch H, 1995. Isolation of the human interleukin 10 promoter. Characterization of the promoter activity in Burkitt's lymphoma cell lines. Cytokine 7: 1-7.

30. Rees LE, Wood NA, Gillespie KM, Lai KN, Gaston K, Mathieson PW, 2002. The interleukin-10-1082 G/A polymorphism: allele frequency in different populations and functional significance. Cell Mol Life Sci 59: 560-569.

31. Crawley E, Kay R, Sillibourne J, Patel P, Hutchinson I, Woo P, 1999. Polymorphic haplotypes of the interleukin-10 5' flanking region determine variable interleukin-10 transcription and are associated with particular phenotypes of juvenile rheumatoid arthritis. Arthritis Rheum 42: 1101-1108.

32. Gibson AW, Edberg JC, Wu J, Westendorp RG, Huizinga TW, Kimberly RP, 2001. Novel single nucleotide polymorphisms in the distal IL-10 promoter affect IL-10 production and enhance the risk of systemic lupus erythematosus. J Immunol 166 : 3915-3922.

33. Fernandez-Reyes D, Craig AG, Kyes SA, Peshu N, Snow RW, Berendt AR, Marsh K, Newbold CI, 1997. A high frequency African coding polymorphism in the N-terminal domain of ICAM-1 predisposing to cerebral malaria in Kenya. Hum Mol Genet 6: 1357-1360.

34. Rook GA, Steele J, Fraher L, Barker S, Karmali R, O'Riordan J, Stanford J, 1986. Vitamin D3, gamma interferon, and control of proliferation of Mycobacterium tuberculosis by human monocytes. Immunology 57: 159-163.

35. Barber Y, Rubio C, Fernandez E, Rubio M, Fibla J, 2001. Host genetic background at CCR5 chemokine receptor and vitamin D receptor loci and human immunodeficiency virus (HIV) type 1 disease progression among HIV-seropositive injection drug users. J Infect Dis 184: 1279-1288.

36. Means TK, Wang S, Lien E, Yoshimura A, Golenbock DT, Fenton MJ, 1999. Human toll-like receptors mediate cellular acti- 
vation by Mycobacterium tuberculosis. J Immunol 163: 39203927.

37. Keane J, Gershon S, Wise RP, Mirabile-Levens E, Kasznica J, Schwieterman WD, Siegel JN, Braun MM, 2001. Tuberculosis associated with infliximab, a tumor necrosis factor alphaneutralizing agent. $N$ Engl J Med 345: 1098-1104.

38. Blackwell JM, Black GF, Peacock CS, Miller EN, Sibthorpe D, Gnananandha D, Shaw JJ, Silveira F, Lins-Lainson Z, Ramos F, Collins A, Shaw MA, 1997. Immunogenetics of leishmanial and mycobacterial infections: the Belem Family Study. Philos Trans $R$ Soc Lond B Biol Sci 352: 1331-1345.

39. Goldfeld AE, Delgado JC, Thim S, Bozon MV, Uglialoro AM, Turbay D, Cohen C, Yunis EJ, 1998. Association of an HLADQ allele with clinical tuberculosis. JAMA 279: 226-228.

40. Selvaraj P, Sriram U, Mathan Kurian S, Reetha AM, Narayanan PR, 2001. Tumour necrosis factor alpha $(-238$ and -308$)$ and beta gene polymorphisms in pulmonary tuberculosis: haplotype analysis with HLA-A, B and DR genes. Tuberculosis (Edinb) 81: 335-341.
41. Roach DR, Briscoe H, Saunders B, France MP, Riminton S, Britton WJ, 2001. Secreted lymphotoxin-alpha is essential for the control of an intracellular bacterial infection. $J$ Exp Med 193: 239-246.

42. Ozaki K, Ohnishi Y, Iida A, Sekine A, Yamada R, Tsunoda T, Sato H, Hori M, Nakamura Y, Tanaka T, 2002. Functional SNPs in the lymphotoxin-alpha gene that are associated with susceptibility to myocardial infarction. Nat Genet 32: 650-654.

43. Carrington M, Nelson G, O'Brien SJ, 2001. Considering genetic profiles in functional studies of immune responsiveness to HIV-1. Immunol Lett 79: 131-140.

44. Al-Sharif FM, Makki RF, Ollier WE, Hajeer AH, 1999. A new microsatellite marker within the promoter region of the MIP1A gene. Immunogenetics 49: 740-741.

45. Saukkonen JJ, Bazydlo B, Thomas M, Strieter RM, Keane J, Kornfeld H, 2002. Beta-chemokines are induced by Mycobacterium tuberculosis and inhibit its growth. Infect Immun 70 : 1684-1693. 